\title{
Los materiales curriculares impresos en educación física: clasificación, usos e investigación
}

José Devís, Carmen Peiró, J. Pere

Miguel Villamón, Luis Antolín y Fernar

(Universitat de Valencia,

\section{Introducción}

El origen y desarrollo de los materiales curriculares corre paralelo al proceso de escolarización de masas que adoptan los Estados desde el inicio de la Modernidad. De entre ellos destacan los libros de texto y otros materiales escritos por ser los más comunes y más extendidos en muchos sistemas educativos (Choppin, 1992; Escolano, 1997; Venezky, 1992). En su actualización y mantenimiento destacan las grandes reformas educativas que han tenido lugar en las distintas naciones-estado. En el caso de España, la reforma de los años setenta del siglo $\mathrm{XX}$ despertó un gran debate teórico y metodológico sobre el tema que condujo a la modernización y consolidación del libro de texto, así como a la diversificación de los recursos pedagógicos de uso escolar. Esta preocupación por los materiales curriculares se vio revitalizada, de nuevo, con la reforma educativa de los años noventa, aunque adaptada a las necesidades y el contexto del momento (García Pascual, 1996; Parcerisa, 1996; Tiana, 1998).

La importancia de los materiales curriculares se refleja en la preocupación que despiertan en los distintos subsistemas de la institución educativa. En una primera instancia resalta la preocupación del centro y el profesorado, ya que son mediadores clave en las actividades docentes y el proceso de enseñanza. Pero también preocupa a la administración educativa a tenor de las reglamentaciones existentes para los distintos tipos de materiales curriculares y la función de control que ejercen sobre el conocimiento escolar. Asimismo, los materiales curriculares son objeto de atención comercial por parte empresas editoriales y las de recursos ma que acaban influyendo decisivamente en ma de concebir y desarrollar el curr (Apple, 1989; Gimeno, 1989 y 1991; Lu1 1992).

En la educación física escolar, los ma curriculares más tradicionales, es decir, 1 dios técnicos tales como las pelotas y las netas, conviven con otros de aparición 1 ciente como los materiales escritos para $\mathrm{e}$ sorado y el alumnado o los medios audior e informáticos. De alguna manera, la apar el uso de los materiales curriculares en la ción física son una consecuencia de la cor ción de nuevas ideas y prácticas de enseî lo largo del tiempo y de la influencia del to social, económico y cultural más amplic y Devís, 1994a).

Sin embargo, el interés y la preocupación materiales curriculares no se ha corresp equivalentemente con su teorización e in ción, tanto dentro del campo general de $k$ dios del curriculum como de los estudios rriculum de la educación física. Por esta realizamos una aproximación teórica a lo riales curriculares en educación física $d \epsilon$ fundamentalmente a su conceptualizacić sificación y uso, y prestando una especic ción a los materiales impresos por ser de 1 pación reciente. Finalizaremos con un r la investigación sobre dichos materiales propuesta de estudio para el contexto espa: hemos elaborado recientemente como gr investigación. 


\section{2. ¿Qué son los materiales curriculares?}

En un sentido amplio, el término 'material curricular' se refiere a cualquier tipo de instrumento $\mathrm{u}$ objeto que se utiliza en las actividades escolares, bien sea con el fin de comunicar contenidos para su aprendizaje o para favorecer y orientar el proceso de enseñanza-aprendizaje. Es decir, son recursos que se utilizan en la planificación, el desarrollo y la evaluación de la enseñanza (Gimeno, 1991; Parcerisa, 1996; Peiró y Devís, 1994a).

Este amplio significado del término no es compartido por otros autores que hacen diferencias entre los 'materiales curriculares', los 'medios' y | los 'recursos'. En este caso, cuando se mencionan los 'materiales curriculares' sólo se hace referencia al material impreso, cuando se habla de 'medios' se alude a los medios audiovisuales y, si se habla de 'recursos' se refiere principalmente a los recursos materiales (San Martín, 1991). Esta forma de abordar el tema parece estar en sintonía con la literatura oficial de la reforma educativa española de los años noventa, puesto que utiliza el término de material curricular para designar, exclusiva y restrictivamente, a los libros de texto y otros materiales escritos que sirven para la elaboración de proyectos curriculares o para orientar la práctica del proceso de enseñanza-aprendizaje (Marchesi y Martín, 1989 y 1991).

Pero esta forma restringida de conceptualizar los materiales curriculares puede esconder la idea de que los términos 'medios' y 'recursos materiales' se apartan del campo de estudios del curriculum por considerarlos simples objetos físicos de carácter neutral. Sin embargo, el uso que se haga de ellos nunca es neutral, ya que se utilizan con unas intenciones, vienen guiados y orientados por ciertos factores y fuerzas socioculturales, y sirven para desarrollar actividades y transmitir valores y conocimientos (intencionada y/o no intencionadamente). El uso de los medios y recursos materiales, al igual que el de los materiales impresos, siempre tiene lugar dentro de un determinado contexto curricular, el cual le confiere un determinado significado educativo. Es más, en última instancia su utilización siempre obedece a una opción moral y política sobre la educación, así como a una determinada opción cultural.

Por todo ello, consideramos que tanto los materiales impresos, como los audiovisuales, informáticos y los recursos materiales son herramientas que sirven para comunicar contenidos $\mathrm{y} / \mathrm{o}$ favorecer y orientar el proceso de enseñanzaaprendizaje. Todos ellos son materiales curriculares que han ido surgiendo y afianzándose con el tiempo como elementos mediadores en el diseño, el desarrollo y la evaluación del curriculum escolar.

\section{Formas de entender los materiales curriculares}

Tal y como ya se ha planteado en otras ocasiones (Martínez, 1991; Peiró y Devís, 1994a), los materiales curriculares en su conjunto se entienden principalmente de dos formas: 1) como instrumentos neutros; y 2) como elementos de experimentación.

\subsection{Los materiales entendidos como instrumentos neutros}

Entender los materiales como instrumentos neutros significa verlos como herramientas que transmiten conceptos e ideas predeterminadas y son utilizados tal y como los presentan las empresas que los elaboran $\mathrm{y}$, obviamente, no presentan posibilidades de comprobación y modificación a partir de la práctica y la experimentación.

La elaboración de este tipo de materiales es encomendada a personas 'expertas' que diseñan, seleccionan y articulan 'correctamente' los contenidos, eligen las actividades 'más adecuadas', y proponen formas de evaluación acordes con 
todo ello. De esta forma el control técnico de la enseñanza queda en sus manos y el profesorado se convierte en el ejecutor de lo que dictaminan los materiales. Es decir, se entienden como una prescripción técnica que debe seguir el profesorado, reduciendo así su capacidad de reflexión y planificación. El ejemplo más claro lo encontramos en el libro de texto del alumnado, tanto por su concepción, como por el diseño, comercialización y forma tradicional de uso (Martínez Bonafé, 1991).

En este sentido, el profesorado se convierte en un mero usuario de la mercancía producida y distribuida por potentes empresas de comercialización (bien en forma de materiales impresos, recursos materiales o audiovisuales), que por su propia lógica de producción y distribución son homogeneizadoras y uniformizadoras del curriculum. De ahí que Apple (1989) los denomine 'materiales a prueba de profesores', es decir, aplicables a cualquier situación, centro o curriculum y por cualquier profesor o profesora. Se considera que los materiales paliarán las posibles deficiencias de preparación de los docentes, independientemente de la formación que tengan, y asegurarán el éxito en el proceso de enseñanza-aprendizaje. Asimismo, se homogeneiza el curriculum de tal forma que se consigue que todo el profesorado alcance los niveles que los expertos han considerado más adecuados, convirtiéndose así en un poderoso mecanismo de control técnico sobre la práctica de la enseñanza.

Esta visión instrumental de los materiales lleva implícita una política curricular o educativa que surge de la desconfianza en la profesionalidad de los docentes y que renuncia a hacer del curriculum un elemento esencial en la transformación de la enseñanza y en la formación del profesorado. Lo que está potenciando es su estancamiento profesional, es decir, una desprofesionalización de los docentes al hacerlos depender de decisiones externas y relegarlos así a meros ejecutantes de unos materiales (Gimeno, 1984). Pero, ade- más, esta desprofesionalización implica la sustitución de las habilidades educativas del profesorado consistentes en elaborar programas y planificar, por las tareas de gestionar y organizar la clase (Kirk, 1990). Para Remillard (2000), los materiales que se comercializan no deberían diseñarse para hablar a través' del profesorado sino para que le hablaran y le permitieran tomar decisiones y adaptarlos a las necesidades contextuales, además de que deberían plasmar las orientaciones pedagógicas que subyacen a las actividades y el curriculum en su conjunto.

Otro aspecto que enfatiza esta visión de los materiales es el individualismo en la práctica de la enseñanza/aprendizaje. El profesor o la profesora dentro de su aula, con su alumnado y sus materiales no tiene que enfrentarse al problema de elaborar y discutir un diseño curricular, ni a coordinar su práctica con los colegas, ni a analizar sus resultados. Simplemente adopta lo que ya viene definido por otros en el libro de texto o cuaderno del alumno. Por otra parte, el alumnado tiene el libro de texto que le infunde seguridad y sabe que en él encontrará todo lo que tiene que aprender y aquello sobre lo que van a evaluarle.

\subsection{Los materiales entendidos como elementos de experimentación}

Desde esta otra perspectiva se entienden los materiales curriculares como una teoría sobre la escuela que no sólo sirven para transmitir conceptos e ideas, sino que son una forma de concebir el desarrollo del curriculum y el trabajo del profesorado y del alumnado (Martínez Bonafé, 1992). Para ello, debemos pensar en la producción y selección de materiales curriculares a partir de la actividad escolar. Como expresa Santos Guerra (1991: 31), nos referimos a "materiales que pueden ser sometidos a la discusión de otros profesionales y que pueden multiplicar las ejemplificaciones surgidas de la experiencia". Los materiales se consideran así, elementos de expe- 
rimentación que pueden comprobarse y modificarse a partir de la práctica y la experiencia permitiendo mejorar la enseñanza. De esta forma, el papel del profesorado no queda reducido a la selección y aplicación sino que se amplía a la elaboración y la evaluación. Esto requiere un esfuerzo de reflexión sistemática, el trabajo en grupo, una discusión compartida, y un enriquecimiento entre el profesorado que los elabora. Es decir, los materiales sirven como elemento de desarrollo profesional, ya que, como manifiesta Contreras (1991: 25), "Un profesor...aprende su oficio trabajando con su herramienta de trabajo, confrontando sus ideas y sus problemas con hipótesis de trabajo que están pensadas para que se desarrollen a partir de las reflexiones y decisiones del profesor, y no aprendiendo instrucciones de uso de paquetes curriculares terminados o recibiendo cursos donde adquirir nuevos conocimientos pedagógicos".

No obstante, cuando hablamos de la elaboración de materiales curriculares por parte del profesorado también debemos tener en cuenta que, aunque hayan sido trabajados en grupo y experimentados en el aula, no serán materiales que se utilicen sin más preámbulos con el alumnado, sino que cada profesor o profesora, en su situación particular, será quien decida la forma de utilizarlos y adaptarlos a sus necesidades pedagógicas y contextuales particulares, y quien tenga la última palabra sobre qué, dónde y cómo utilizar los materiales, la organización de las clases, etc. Se trata del conocimiento experto del profesorado sobre la selección, adaptación, creación y evaluación de los materiales que debería ser altamente valorado por parte de la administración educativa.

Los materiales deberían sugerir un modo de trabajo, de selección y organización del conocimiento y servir como ejemplo de estrategias de calidad que provocaran en el profesorado la emisión de juicios comprometidos sobre su tarea, la reflexión y el debate. Es decir, deberían ser abiertos y flexibles, de tal forma que permitieran al profe- sorado investigar en su contexto práctico concreto para poder completarlos y modificarlos.

Así pues, los materiales deberían ofrecer, por una parte, la posibilidad de ser estructurados y adaptados por el profesorado dependiendo de cada contexto en particular y, por otra, la de evaluar el impacto que producen en las situaciones concretas del aula. Esta forma de concebir los materiales responde a un proyecto curricular centrado en el dominio de habilidades procesuales más que al aprendizaje de contenidos específicos, y a un modelo curricular de proceso, en el que los profesores y profesoras adquieren mayor autonomía mediante la reflexión sobre su propia práctica.

\section{Los materiales curriculares en la educación física escolar: una clasificación}

En la literatura general sobre el tema encontramos diversas formas de clasificar los materiales curriculares, tantas como criterios puedan utilizarse para ello. Cabero (1990) ha ordenado distintas clasificaciones y taxonomías atendiendo a criterios tales como: a) los sentidos implicados; b) el grado de realismo del material; c) el lenguaje; d) la relación con el profesorado; e) el momento histórico de aparición; f) el sistema de catalogación; y g) las funciones didácticas de los materiales. Para este autor, la mayoría de clasificaciones se han fundamentado en enfoques conductuales y han servido de poca ayuda a la actividad docente del profesorado. Recientemente se han seguido criterios o enfoques cognitivistas como ocurre con la clasificación de Zabala (1990) que menciona los criterios siguientes: a) el nivel de concreción curricular, tal y como señala la reforma curricular española de los años noventa; b) la intencionalidad o función del material (orientar, proponer, ejemplificar, etc.); c) el tipo de contenidos en el que incide prioritariamente cada material; y d) el medio o soporte en el que se encuentra el material (papel, electrónico, audiovisual, etc.). 
Algunas clasificaciones generales como la realizada por la UNESCO han llegado a incluir los medios de enseñanza de la educación física como una categoría específica de materiales junto a los manuales y libros, los medios para la enseñanza científica, los medios para la enseñanza técnica y profesional, $y$ los medios audiovisuales e informáticos (en Parcerisa, 1996). Sin embargo, la evolución de la educación física como asignatura escolar junto a los avances producidos en los materiales $y$ las necesidades de cada momento han resultado actualmente en una amplia variedad de materiales que hemos agrupado en: a) los materiales impresos; b) los recursos materiales; $y$ c) los medios audiovisuales $\mathrm{e}$ informáticos (ver tabla 1).

Esta clasificación, como cualquier otra, puede resultar problemática por dejar fuera algún tipo de materiales o porque no es mutuamente excluyeme entre sus categorías. Pensemos que en la realidad de la enseñanza los materiales no aparecen aislados sino que, a menudo, se relacionan entre sí. Incluso un material concreto extraído de la prensa diaria puede considerarse como material impreso, al referirnos a los artículos escritos, o como medio audiovisual al referirnos a sus imágenes. Por otra parte, tan importante o más que la clasificación es el uso que se haga de ellos, por eso nuestro comentario de los materiales lo acompañaremos de reflexiones sobre su uso.

Si bien los materiales impresos son los más utilizados dentro del sistema escolar (Área, 1994), en el área de educación física, como ya hemos mencionado antes, han sido los recursos materiales (pelotas, colchonetas, etc.) los que se han erigido a lo largo del tiempo como los materiales curriculares dominantes. Las prácticas que éstos representan son una selección de la cultura física que condiciona las clases de educación física. En unos casos contribuyen a homogeneizar los contenidos de la asignatura (p. ej. los materiales de los deportes estándar) y, en otros, se pretende introducir prácticas nuevas o revitalizar otras vie- jas (p. ej. los materiales alternativos o tradicionales). Sin embargo, el uso que se haga de unos y otros puede cambiar radicalmente el sentido con el que se concibieron inicialmente, esto es, puede utilizarse material estándar de forma no convencional y material alternativo para introducir prácticas que enfatizan un curriculum tradicional en la forma de enseñarlo y en la interacción profesor-alumno (Peiró y Devís, 1994a).

La incorporación de los medios audiovisuales e informáticos a la enseñanza de la educación física supone un replanteamiento global de los procedimientos didácticos, del papel del profesorado y de la acción docente, así como cambios profundos de actitudes que impliquen a toda la comunidad educativa. Sin embargo, debemos recordar que, en sí mismos, no tienen capacidad didáctica ninguna y su uso indiscriminado e improvisado les convierte en elementos inútiles e incluso perjudiciales (San Martín, 1986). Los medios audiovisuales e informáticos pueden aportar fluidez a las clases y lograr un clima de continuidad pedagógica, pero también pueden utilizarse como relleno o recompensa al buen comportamiento del alumnado. Volvemos a insistir que, dependiendo de cómo se utilicen, pueden fomentar el conformismo social, facilitar el aprendizaje o ayudar a analizar críticamente las actividades físicas y deportivas para defenderse del poder e influencia que ejercen los medios de comunicación en la cultura física de la sociedad. Además del uso de los medios audiovisuales como recurso didáctico para el aprendizaje de alguna actividad física, también sirve para reflexionar críticamente sobre el fenómeno sociocultural de la educación física y el deporte. Así, por ejemplo, dentro de nuestra asignatura podemos cuestionar ciertos valores que se transmiten a través de la publicidad y de algunos programas televisivos (el culto a la esbeltez en las mujeres y del cuerpo atlético en los hombres y sus relaciones con el ejercicio físico, el encasillamiento de actividades y deportes según el género, la agresividad como aspecto inherente al deporte, etc.). Asimismo, 
podemos facilitar que el alumnado analice y haga explícitos esos tópicos y valores y los relacione con otros aspectos socioculturales, económicos y políticos que inciden directamente en la educación física y el deporte (ver p. ej. Duran, 1999;
Gutiérrez y Montalbán, 1994; Mes

Cuando mencionamos los materia dentro de la educación física, gen€ asociamos con materiales vinculadc

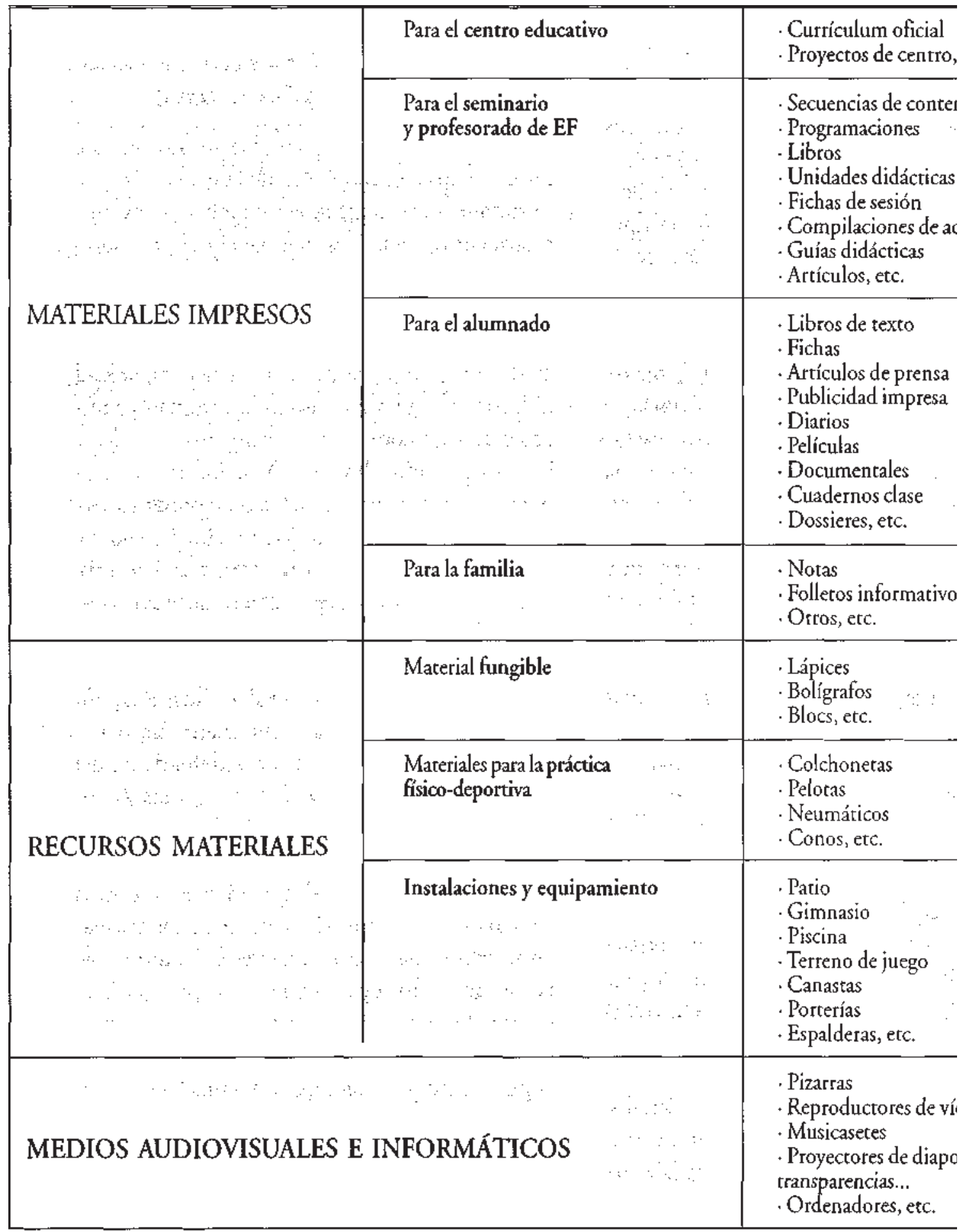

Tabla 1 - Clasificación de los materiales curriculares en educación fisica (a partir Devis, 1994a) 
rado, bien en forma de libros pedagógicos y didácticos, carpetas de fichas u otro tipo de material. En menor medida pensamos en los materiales dirigidos al alumnado. Con ellos podemos favorecer la relación entre las clases teóricas y las prácticas dentro de la asignatura, pero también podemos acusar la separación entre la teoría y la práctica, especialmente si toman la forma de libro de texto. Según quién, cómo y desde qué supuestos teóricos diseñe estos materiales, así como el uso que haga de ellos, podrá facilitarse la conexión o la separación entre la teoría y la práctica (Devís, 1996; Peiró y Devís, 1994b).

Otro tipo de materiales impresos con los que apenas pensamos son los dirigidos a la familia. Con ellos pretende implicarse y hacer partícipe a la familia del proceso educativo porque sugieren actividades de apoyo en casa, relacionadas con el contenido que vaya a desarrollarse, o bien tratan de vincular a las familias en actividades del centro especialmente relacionadas con la educación física.

\section{Los materiales curriculares impresos para el alumnado y sus intenciones de uso}

La noción de material curricular que adoptamos los autores de este artículo deriva del discurso presentado en el apartado anterior. Es decir, entendemos como material curricular impreso todo documento que se encuentra en soporte papel, audiovisual o informático y codifica su información mediante un lenguaje textual que puede combinarse con representaciones ¡cónicas. Generalmente los materiales curriculares impresos son textos, representaciones de textos o adaptaciones de texto en combinación con iconos o imágenes (Área, 1994). El texto es una forma esencial de elaboración, almacenamiento y recuperación de información, en definitiva, una forma de tratamiento del conocimiento cultural y escolar. Así, por ejemplo, un documental sobre las olimpiadas representa o adapta un guión, es decir, un texto impreso en soporte audiovisual que puede se utilizado como material informativo para el profesorado o para su uso directo con el alumnado.

A partir de esta definición y de algunos trabajos anteriores (Devís, 1998; Peiró, 2000), analizamos a continuación los materiales curriculares impresos para el alumnado de educación física y exploramos las intenciones de uso y su vinculación con las prácticas docentes.

\section{El material informativo}

El material informativo es aquél que esencialmente proporciona conocimiento teórico de diversa índole al alumnado. Tipos de materiales informativos pueden ser los libros o partes de libros, artículos de revistas o de prensa o los documentales sobre determinados temas físicodeportivos. En cualquier caso, si pensamos en estos materiales como elementos de experimentación no podemos limitarnos a utilizar, sin más, los libros o artículos elaborados por otras personas. Deberíamos realizar algunas modificaciones, selecciones o simplemente enfocar la lectura del mismo acompañando el texto de preguntas o temas de especial interés para la asignatura (ver material de la figura 1).

Naturalmente, el material más acorde con la idea de experimentación es el elaborado por el propio profesorado con la intención de adaptarlo especialmente a las circunstancias y características de su alumnado. Este material también puede verse acompañado de preguntas o reflexiones que conecten con algún tipo de experiencia física del alumnado, es decir que traten, en definitiva, de vincular la información presentada con vivencias del alumnado y favorecer así un aprendizaje más significativo. Asimismo, este tipo de material puede plantearse de tal manera que la información facilitada y las preguntas formuladas introduzcan al alumnado en los aspectos problemáticos y controvertidos vinculados a la actividad física, el deporte, el cuerpo, la cultura física, etc. 


\section{El corazón nos informa}

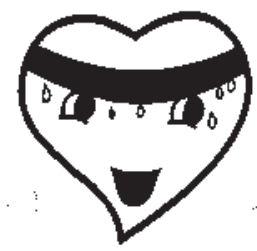

Soy el corazón y te voy a dat información

sobre el ejercicio y tu capacidad para hacerlo

Me encargo de bombear sangre a todo el cuerpo y en ella van las

sustancias que te darán energía para moverte

Cuando más intenso es el ejercicio más

energía necesitas y más rápido tengo que latir

Para averiguar la intensidad del ejercicio que haces, debes

saber tomarte el pulso: en la muñeca, en el cuello, en el corazón...

Cuando comiences a realizar ejercicio debes de aumentar

lentamente las pulsaciones. Al finalizar el mismo no te pares, disminuye la intensidad

para que desciendan las pulsaciones poco a poco

\section{Piensa y contesta:}

Las pulsaciones del corazón las llamamos también.

En qué partes del cuerpo podemos tomarnos el pulso?.

Fuente: Fraile (coord.) (1996)

Figura 1. Material informativo para la enseñanza primaria

\section{El material de conexión teoría-práctica}

Este tipo de material es fundamental en la educación física porque permite conjugar conceptos teóricos (saber qué) con procedimientos (saber cómo) e incluso actitudes (saber ser). Esta conjunción resulta útil e interesante si tenemos en cuenta que estamos ante una asignatura eminentemente práctica con importantes limitaciones horarias que dificultan un desarrollo curricular adecuado.

Este material, que resulta ideal utilizarlo durante el desarrollo de alguna actividad física, favorece que el conocimiento teórico se experimente, aprenda y vincule a la práctica física, permitiendo que el alumnado reflexione y comprenda los conceptos en la práctica $y$ sobre la práctica (ver, por ejemplo, el material de la figura 2). Así, un aprendizaje experiencial resultará más significativo, enriquecedor y es más probable que facilite una mejor asimilación e integración de los conocimientos.

Como ocurre con el material informativo, el de conexión teoría-práctica puede tomarse de algún otro libro y publicación o ser de elaboración propia, pero en cualquier caso debe dirigirse a con- 
textos particulares de enseñanza, circunstancia que exige la realización de diversas adaptaciones.

\section{El material de recopilación}

El material de recopilación, como el presentado en la figura 3 , recoge experiencias, con- tenidos y conclusiones a las que llega el alumnado en las sesiones de clase y, generalmente, se utilizan al final y/o después de éstas con el fin de repasar, comprender, profundizar y reflexionar sobre aspectos o temas de la educación física. Asimismo, este material puede permitir detectar las experiencias y los conocimientos previos que tiene el

\section{Tema: La frecuencia respiratoria}

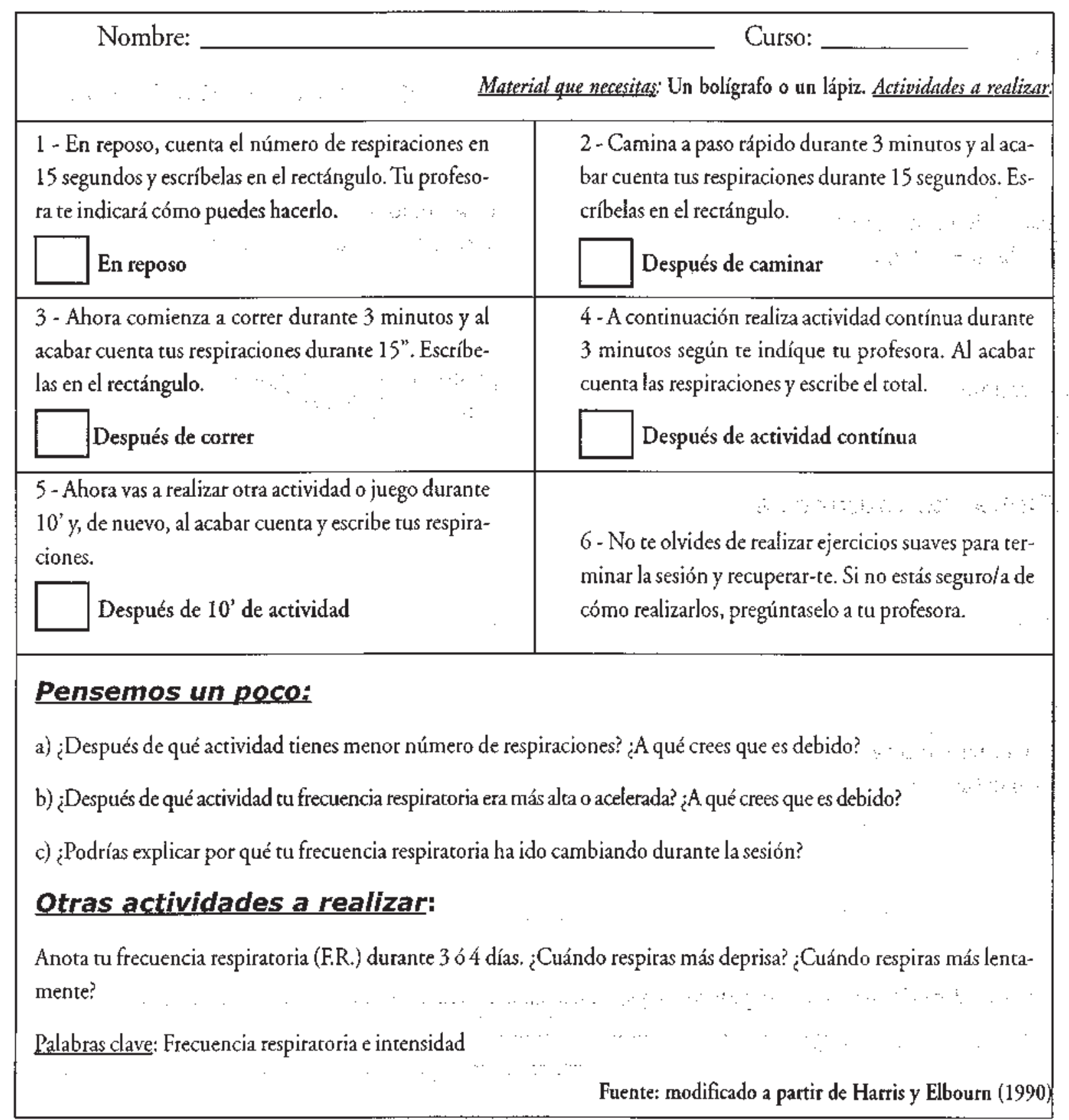

Figura 2. Materlal de conexión teoría-práctica para la enseñanza primaria 
alumnado sobre los temas propuestos y motivarle a aprender más sobre ellos. Se trata, en definitiva, de favorecer la reflexión y la comprensión de lo que se hace en clase y ampliar el conocimiento.

Ejemplos de materiales de recopilación pueden ser fichas (en las que el alumnado responde a una serie de preguntas), dossieres (que recopilan determinados tipos de ejercicios y explicaciones de ellos, o recortes de revistas, periódicos, etc. sobre un tema determinado), y también podrían considerarse material de recopilación los diarios del alumnado y los cuadernos de clase. El diario, por ejemplo, puede presentar un formato elaborado por el profesorado para que lo rellene el alumnado o bien puede plantearse de tal manera que sea el alumnado quien lo elabore, estimulando así también la creatividad, si bien esto dependerá del desarrollo y madurez de los niños y de su experiencia en este tipo de habilidades de redacción, entre otras cuestiones.

\section{El material de evaluación}

Este tipo de material permite obtener información del proceso de enseñanza-aprendizaje y, a partir de ella, poder realizar las modificaciones $\mathrm{y}$ ajustes pertinentes. Este material permite al alumnado descubrir el significado de las actividades que plantea el profesorado, identificar las cuestiones que se consideran importantes y los conocimientos que ha adquirido.

Ejemplos de este tipo de material puede ser la elaboración de una ficha de autoevaluación donde aparezcan reflejados los objetivos de una unidad didáctica concreta y el alumnado ha de señalar si va mejorando o ha conseguido cada uno de los objetivos establecidos. También una prueba escrita, a modo de examen, sería un ejemplo de este tipo de material, pero con el propósito de plantear preguntas que vayan más allá de la mera constatación de determinados conceptos aprendidos por el alumnado y que, más bien, se dirijan a la emisión de respuestas razonadas

\section{Ficha del alumno/a}

Nombre: Curso:

Tengo años

La fecha de hoy es: .

Voy a dibujar la actividad que más me ha gustado de la clase de hoy, después la pintaré y pondré un nombre a mi dibujo.

Esta actividad es la que más me ha gustado porque

Fuente: Fraile (coord.) (1996)

Figura 3. Material de recopilación para la enseñanza primaria 


\section{Pierda 8 kilos en un mes}

Dibujo de una

....mujer joven,

esbeita $y$ blanca.

¡ GRATIS! Una magnífica camiseta para usied

Teléfono:

Dirección:

Cupón para una prueba sin compromiso

\section{ANÁLISIS DEL MENSAJE ESCRITO}

1. Titular y ventajas que ofrece el producto:

2. ¿Por qué quiere destacar la rapidez de su efecto? ¿Por qué no hace falta esfuezo?

3. Si fuera cierto el anuncio, ̨̧crees que sería saludable perder $8 \mathrm{kgs}$. tan rápidamente? ¿Por qué?

4. ¿Regalan algo? ¿Por qué?

5. ¿El anuncio dice algo sobre llevar una dieta equilibrada o realizar algún ejercicio físico?

6. ¿Crees que se puede conseguir un cuerpo como el de la fotografia con ese producto?

\section{ANÁLISIS DE LA IMAGEN}

1. ¿La persona de la fotografía es una mujer o un hombre?

2. ¿Es un niño/a, joven o anciano/a? ¿'De qué raza es la persona?

3. ¿Por qué crees que han elegido esta persona y no oura?

4. ¿El modelo de belleza corporal actual ha sido siempre el mismo a lo largo de la historia? ¿Pregunta a rus padres o abuelos?

5. ¿Qué ocurre con las personas que no tienen el modelo de cuerpo de la persona de la fotografía?

\section{ANÁLISIS DEL PRECIO}

1. Precio inicial:

2. Precio real:

\section{OPINIÓN PERSONAL}

\section{Figura 4. Material crítico}


que atiendan al porqué, el para qué y el cómo de los contenidos. Además, sería conveniente que los conocimientos reflejados en este material fueran posteriormente comentados con el alumnado, a modo de feedback, con el fin de aclarar dudas, así como reforzar y consolidar el aprendizaje.

\section{El material para promover la actitud crítica}

Estos materiales curriculares son fundamentales para ayudar a la reflexión crítica acerca de diversos aspectos concernientes a la educación física. Con la elaboración y utilización de este tipo de material se pretende ayudar al alumnado a desenmascarar una serie de valores, creencias y distorsiones relacionadas con cuestiones sociales que van más allá de la actividad física (ver material de la figura 4). Algunos ejemplos son los materiales para analizar anuncios publicitarios impresos y televisivos, dibujos animados relacionados con prácticas físicas, artículos de revista o periódico, etc. También pueden elaborarse cuentos o historias ficticias que pueden escenificarse o leerse y que van acompañadas de preguntas para la reflexión.

No obstante, si bien la utilización de este tipo de materiales es importante, también lo es la actitud y los comentarios del profesorado en las clases que tengan relación con cuestiones controvertidas. $\mathrm{Y}$ es que este tema de la promoción de la actitud crítica es muy delicado puesto que está vinculado a aspectos morales y éticos. Supone estar muy sensibilizado con él y requiere una reflexión previa por parte del profesorado de qué supone utilizar este tipo de materiales y tener siempre presente las consecuencias de las actuaciones, los comentarios y las actitudes, de tal manera que se sea coherente con una perspectiva para la cual se crean y utilizan estos materiales.

\section{La combinación de diversos materiales curriculares}

Los materiales que hasta ahora se han presentado ilustran un tipo de material concreto y específico y se han presentado de manera aislada, pero los materiales también pueden utilizarse mediante combinaciones más complejas para distintas intenciones u objetivos. Así, por ejemplo, algunos de los materiales de recopilación, sobre todo el diario y el cuaderno del alumno/a, también pueden utilizarse como material de evaluación, puesto que en estos materiales el alumnado no sólo recoge los contenidos conceptuales y procedimentales que ha aprendido, sino que generalmente expresa y refleja aspectos de tipo afectivo como, sensaciones, experiencias, cuestiones relativas a sus relaciones con compañeros y/o actitudes ante determinadas situaciones. Estos datos deberían ser considerados, y son esenciales, para que el profesorado pueda evaluar (y conocer) individualmente a cada alumno/a, así como evaluar el propio planteamiento y proceso de enseñanza-aprendizaje.

En muchas ocasiones, los diarios sirven para dar voz a aquellos alumnos que en clase pasan desapercibidos, como si de alumnos invisibles se tratara, pero que gracias al diario pueden reflejar, y el profesorado puede conocer, parte del proceso interno y lo que ha significado la asignatura para él o ella, además de saber lo que ha aprendido.

\section{La investigación sobre materiales curriculares impresos}

La importancia de los materiales curriculares en el desarrollo del curriculum y la existencia de medios tecnológicos para su producción y difusión han fomentado la elaboración de materiales comerciales y una extensa literatura sobre el tema (Johnsen, 1996; Woodward, Elliot y Nagel, 1988). Sin embargo, es curioso observar la escasa atención prestada a los materiales desde la investigación educativa, como así lo manifiestan al- 
gunas de las revisiones realizadas (Abraham y Rojas, 1997; Lumsdaine, 1988; Weinbrenner, 1992). Si bien los orígenes de la investigación sobre el libro de texto y otros materiales curriculares se sitúan en el contexto educativo norteamericano del primer cuarto del siglo XX (Escudero, 1983), la mayoría se han centrado especialmente en el estudio de los medios audiovisuales (Cabero, 1990; Castaño, 1994; Tiana, 1998).

Las primeras investigaciones específicamente dirigidas al libro escolar y otros materiales impresos fueron de tipo evaluativo y formaron parte de proyectos de desarrollo y cambio curricular (Fullan, 1983). Estaban influidas por una visión instrumental de los materiales porque trataban de comprobar la fidelidad y correspondencia entre las metas de los programas y el proceso de aplicación de los nuevos textos y materiales. Posteriormente, la investigación se ocupó del papel mediador y transformador del profesorado en el desarrollo del curriculum. Así surgieron investigaciones centradas en las opiniones, intereses, percepciones y expectativas del profesorado sobre los libros y materiales curriculares (BenPeretz y Tamir, 1981; Connelly y Ben-Peretz, 1980; Leithwood et al, 1976).

Otro grupo de investigaciones, surgidas por influencia de la nueva sociología de la educación de finales de los años setenta, se centraron en el análisis ideológico del conocimiento escolar contenido en los libros de texto (Apple, 1982 y 1989). En esta época aparecen las primeras investigaciones españolas sobre el libro de texto y los materiales curriculares. Emergieron en conexión con el proceso de institucionalización y expansión de la investigación educativa y recogían las opiniones del profesorado sobre libros y materiales (Tiana, 1998). En los años setenta y principios de los ochenta también crece el interés por el análisis y evaluación de los libros de texto, aunque desde un punto de vista técnico que sirviera para su elaboración comercial (p.ej. Bernard,
1974; López, 1982; Velasco y Pérez, 1977). Fue a finales de los ochenta cuando las investigaciones se centraron en el análisis de los libros de texto para desentrañar los mensajes ocultos relativos al racismo, el sexismo y otras manifestaciones ideológicas (p. ej. Calvo, 1989; Garreta y Careaga, 1987; Moreno, 1987) y, en los noventa, el análisis del conocimiento escolar se aborda desde la óptica particular de distintas disciplinas escolares (p. ej. Argibay, Celorio y Celorio, 1990 ó 91). Por entonces aparecen también algunas tesis doctorales que analizan el libro de texto y lo relacionan con el modelo curricular de la reforma (García Pascual, 1996; Parcerisa, 1995) y con la regulación de la profesionalidad docente (Cantarero, 2000).

La evolución sufrida por la investigación realizada en el-ámbito de la educación física es similar al proceso general descrito anteriormente. De la evaluación de proyectos curriculares de la asignatura se pasó al análisis del sexismo en los libros de texto (Browne, 1990; Hildreth, 1981) o alas repercusiones de los paquetes curriculares en el trabajo del profesorado y el desarrollo conceptual de la educación física (Dewar, 1985; Kirk, 1990; Placek, 1989; Schempp, 1982). En el contexto educativo español, el libro de texto y otros materiales escritos empezaron a constituir un elemento de preocupación de los profesionales de la educación física durante la segunda mitad de la década de los años ochenta (Muñoz, Olivera, Pérez, Sánchez y Sainz, 1988). La relevancia que ha tenido la línea de investigación española centrada en el análisis y evaluación del libro de texto se ha visto reflejado en las primeras propuestas de análisis dirigidas al estudio del sexismo (Ribas y Guerrero, 1994) y los primeros análisis empíricos de libros de texto en la educación física escolar (Delgado, Barrera y Medina, 1992; Peiró y Devís, 1994b).

Desde nuestro punto de vista, esta línea de investigación en educación física ofrece nuevas posibilidad es para la mejora de libros y materiales im- 
presos, no sólo de sus aspectos formales y técnicos, sino de los aspectos ideológicos y éticos que en ellos se encuentren implícitos (p. ej. sexismo, igualitarismo, rendimiento, recreacionismo, culto a la delgadez o mesomorfismo). Se preocupa especialmente por cuestiones tales como, qué intereses encubren los materiales, qué valores transmiten y qué condiciones, relacionadas con el curriculum oculto, influyen en la utilización de estos materiales. Digamos que la mejora de materiales y contenidos está relacionada con las actitudes y valores que, a pesar de especificarse en el curriculum oficial español, al profesorado le resulta difícil trasladar a la práctica.

Durante los últimos años, otra línea de investigación reclama la necesidad de evaluar los libros y materiales dentro de los contextos curriculares y en situaciones de uso real (Área, 1991; Kirk, 1990; Santos, 1991; Venezky, 1992). Es decir, trata de saber qué hace el profesorado con los materiales curriculares durante la enseñanza. Aunque el uso de materiales es un tema presente en la bibliografía específica, la escasa investigación empírica se ocupa de recoger información mediante encuesta para conocer cómo utiliza el profesorado el libro de texto en las clases (Rodríguez Diéguez et al, 1998) o de recoger información mediante la observación directa del uso de los libros y materiales en la planificación y en el desarrollo curricular (Área, 1986 y 1991; Kirk, Gore y Colquhoun, 1989). Este último tipo de investigación resulta muy pertinente para examinar el papel que juegan los materiales impresos en la planificación, el proceso de instrucción, las decisiones del profesorado y las estructuras comunicativas de la clase, así como su relación con el resto de componentes curriculares de la educación física. Además, permiten conocer la influencia que ejercen los materiales en la conformación de un determinado modelo de enseñanza y racionalidad curricular.

\section{Comentarios finales: una propuesta de investigación para el contexto español}

Los materiales curriculares impresos son elementos tradicionales de la tecnología educativa en España, pero se han visto impulsados dentro de la educación física escolar desde la reforma de la década de los años noventa del siglo XX. Esto resulta especialmente novedoso por lo que respecta a los materiales dirigidos al alumnado (libros de texto y otros materiales impresos), circunstancia que aconseja evaluar cómo está introduciéndose este fenómeno en nuestras escuelas. Por ello, un grupo de profesores y estudiantes vinculados a la Unidad de Investigación de 'Teoría y Pedagogía de la Actividad Física y el Deporte' de la Universitat de Valencia, hemos iniciado un programa de investigación sobre el uso de materiales curriculares impresos en educación física. Lo hemos dirigido al profesorado porque la literatura lo señala como el colectivo del que dependen las decisiones relativas a la selección, elaboración en su caso, y forma de uso del material escrito durante las clases (p. ej. Área, 1991, 1994; Parcerisa, 1996). El ámbito de desarrollo es la Educación Secundaria Obligatoria (ESO: edades entre los 12 y 16 años) de la Comunidad Autónoma Valenciana por tratarse del nivel educativo y el contexto geográfico con el que está especialmente vinculado nuestro trabajo universitario.

El programa de investigación consta de dos grandes fases. Una primera que permite conocer ampliamente el uso de los materiales en la Comunidad Autónoma Valenciana a partir de lo que dice una muestra del profesorado de educación física. Una segunda fase que permite conocer en profundidad el uso de los materiales durante el propio desarrollo del curriculum a partir de la selección de casos que surjan de los perfiles de uso identificados en la fase anterior. La primera fase responde a un estudio descriptivo de carácter cuantitativo, por lo tanto sustentado por una epistemología objetivista, y derivado del análisis 
de un cuestionario elaborado al efecto. La segunda es un estudio cualitativo sustentado por una epistemología subjetivista y derivado del estudio de varios casos de profesores y profesoras correspondientes a las concepciones de uso encontradas en la primera fase.

Este programa pretende estar en sintonía con las líneas recientes de investigación sobre materiales curriculares que se han expuesto en el apartado anterior y pretende servir de ayuda para: a) conocer el papel de los materiales impresos en la planificación, desarrollo y evaluación del curriculum de la educación física, es decir, conocer criterios de selección, elaboración y uso, así como niveles, preferencias y concepciones de uso entre el profesorado; b) identificar formas de uso de dichos materiales que sean indicativos de alta calidad de la enseñanza; y c) orientar la formación inicial y permanente del profesorado a partir de un conocimiento empírico sobre un tema del que se habla y escribe mucho, pero se investiga poco.

\section{Bibliografía}

Abraham, M. y Rojas, A. (1997) La investigación educativa en Latinoamérica. Revista de Educación, 312, pp. 21-42.

Apple, M.W. (1982) Curricular form and the logic of technical control: building the possessive individual. En M.W. Apple (ed.) Cultural and economic reproduction in education: Essays on class, ideology and the State, Routledge \& Kegan Paul, London, pp. 247-274.

Apple, M.W. (1989) Maestros y textos. Una economía política de las relaciones de clase y de sexo en educación. Paidós/MEC. Barcelona.

Área, M. (1986) Un estudio sobre las decisiones docentes de uso del libro de texto en situaciones de enseñanza. En L.M. Villar (ed.) Pensamientos de los profesores y toma de decisiones, Servicio de Publicaciones de la Universidad de Sevilla, Sevilla, pp. 422-435.

Área, M. (1991) Los medios, los profesores y el currículo. Sendai. Hospitalet de Llobregat.

Área, M. (1994) Los medios y materiales impresos en el curriculum. En J.M ${ }^{\text {a }}$ Sancho (coord.) Para una tecnología educativa, Horsori, Barcelona, pp. 85-113.

Argibay, M.; Celorio, G. y Celorio, J.J. (1990) Análisis de los libros de texto de Ciencias Sociales. Una visión desde la educación para el desarrollo. Colección Documentos 2. Generalitat Valenciana. Valencia.

Ben-Peretz, M. y Tamir, P. (1981) What teachers want to know about curriculum materiais. Journal of Curriculum Studies, 13, 45-54.

Bernard, J. (1974) Guía para la valoración de textos escolares. Teide. Barcelona.

Browne, J. (1990) Gender bias in physical education textbooks. ACHPER National Journal, 127, 47.

Cabero, J. (1990) Análisis de los medios de enseñanza. Aportaciones para su selección, utilización, diseño e investigación. Alfar. Sevilla.

Calvo, T (1989) Los racistas son los otros. Gitanos, minorias y derechos humanos en los textos escolares. Editorial Popular. Madrid.

Cantarero, J.E. (2000) Materiales curriculares y descualificación docente. Análisis interpretativo de las estrategias a través de las que el libro de texto regula el trabajo del profesorado. Tesis doctoral. Universitat de Valencia.

Castaño, C. (1994) La investigación en medios y materiales de enseñanza. En J.M ${ }^{\mathrm{a}}$ Sancho (coord.) Para una tecnología educativa, Horsori, Barcelona, pp. 269-295.

Choppin, A. (1992) Les manuels scolaires. Historie at actualité. Hachette. París. 
Connelly, EM. y Ben-Peretz, M. (1980) Teachers' roles in the using and doing of research and curriculum development. Journal of Curriculum Studies, 12, 95-107.

Contreras, J. (1991) El curriculum como formación. Cuadernos de Pedagogía, 194, 22-25.

Delgado, M.A. Barrera, J. y Medina, J. (1992) Análisis del libro de texto en la enseñanza de la educación física. Habilidad Motriz, 1, 11-18.

Devís, J. (1996) Educación física, deporte y curriculum. Visor. Madrid.

Devís, J. (1998) La salud en la educación física escolar: materiales curriculares para el alumnado de enseñanza primaria. En M. Villamón (dir.) $L a$ educación física en el curriculum de primaria, Conselleria de Cultura, Educació i Ciencia, Generalitat Valenciana, Valencia, pp. 367-388.

Dewar, A. (1985) Curriculum development and teachers' work: the case of the Basic Stuff Series in physical education. En M. Carnes (ed.) Proceedings of the Fourth Conference on Curriculum Theory in Physical Education, University of Georgia, Athens, pp. 158-167-

Duran, J. (1999) Deportes y medios de comunicación: una propuesta educativa. . En M.L. Martín y J.C. Narganes (dirs.) La educación física en el siglo XXI, Fondo Editorial de la Enseñanza, Jerez, pp. 327-352.

Escolano, A. (1997) Libros para la escuela. La primera generación de manuales escolares. En A. Escolano (dir.) Historia ilustrada del libro escolar en España. Del Antiguo Régimen a la Segunda República, Fundación Germán Sánchez Ruipérez-Pirámide, Madrid, pp. 19-46.

Escudero, J.M. (1983) La investigación sobre los medios de enseñanza: revisión y perspectivas actuales. Enseñanza, 1, 87- 119.

Fraile, A. (1996) (coord.): Actividad física y salud en la escuela. Junta de Castilla y León. Valladolid.

Fullan, M. (1983) Evaluating program implementation: What can be learned from Follow-through. Curriculum Inquiry, 13, 215227.

García Pascual, E. (1996) Libros de texto y reforma educativa: Un análisis de los textos escolares de educación primaria. Tesis doctoral. Universidad de Zaragoza.

Garreta, N. y Careaga, P. (1987) Modelos masculino y femenino en los textos de EGB. Ministerio de Cultura: Instituto de la Mujer. Madrid.

Gimeno, J. (1984) Prólogo. En L. Stenhouse Investigación y desarrollo del curriculum, Morata, Madrid, pp.9-24.

Gimeno, J. (1989) El curriculum: una reflexión sobre la práctica. Morata. Madrid.

Gimeno, J. (1991) Los materiales y la enseñanza. Cuadernos de Pedagogia, 194, 10-15.

Gutiérrez, M. y Montalbán, A. (1994) ¿Qué valores transmite la serie deportiva de dibujos animados Campeones? Revista Española de Educación Física y Deportes, 1 (2) 26-33.

Harris, J y Elbourn, J. (1990): Action for heart health. Centre for Exercise and Health. Loughborough.

Hildreth, K. (1981) Sexism in elementary physical education literature: a content analysis. Microficha. University of Oregon.

Jonhsen, E.B. (1996) Libros de texto en el calidoscopio. Pomares. Barcelona.

Kirk, D. (1990) School knowledge and the curriculum package-as-text. Journal of Curriculum Studies, 22, 409-425.

Kirk, D.; Gore, J. y Colquhoun, D. (1989) Teachers' use of the daily physical education program and the problem of fitness development. ACHPER National Journal, 124, 23-27.

Leithwood, K.A.; Clipsham, J.S.; Maynes, F. y Baxter, R. (1976) Curriculum change at the systems levei: a four-year mathematics project. Curriculum Theory Network, 5, 219-245. 
López, N. (1982) Cómo valorar textos escolares. Cincel. Madrid.

Lundgren, U.P. (1992) Teoría del curriculum y escolarización. Morata. Madrid.

Lumsdaine, A.A. (1988) Instruments and Media of Instruction. En N.L. Gage Handbook of Research on Teaching. UMI. Michigan, pp.583682.

Marchesi, A. y Martín, E. (1989) Reforma de la enseñanza, reforma del curriculum. Cuadernos de Pedagogía ,168.

Marchesi, A. y Martín, E. (1991) Lo que dice el MEC sobre materiales. Cuadernos de Pedagogía, 194, 46-48.

Martínez Bonafé, J. (1991) Proyectos curriculares y práctica docente. Diada. Sevilla.

Martínez Bonafé, J. (1992) Siete cuestiones y una propuesta. Cuadernos de Pedagogía, 203, 8-13.

Mesa, R. (1999) El profesorado de educación física ante el consumismo y los mensajes emitidos por los medios. En M.L. Martín y J.C. Narganes (dirs.) La educación física en el siglo XXI, Fondo Editorial de la Enseñanza, Jerez, pp. 353-362.

Moreno, A. (1987) El arquetipo viril protagonista de la historia. La Sal. Barcelona.

Muñoz, E.; Olivera, J.; Pérez, J.I.; Sánchez, M. y Sainz, R. (1988) El libro de texto en la educación física de las enseñanzas medias. En M. Chivite y otros Aspectos didácticos de educación física 2, ICE, Universidad de Zaragoza, Zaragoza, pp. 37-78.

Parcerisa (1995) Eis materiais curriculars a l'ensenyament Primari. Análisi deis materiais curriculars editats en suport paper utilitzats a l'Educado Primaria del nou sistema educatiu. Tesis doctoral. Universitat de Barcelona.

Parcerisa, A. (1996) Materiales curriculares. Cómo elaborarlos, seleccionarlos y usarlos. Graó. Barcelona.

Peiró, C. (2000) La educación física para la salud en la escuela: modelos teóricos y materiales curriculares. En M.L. Martín; S. León y J.C. Narganes (dirs.) Educación fisica y salud, FETEUGT, Jerez, pp. 223-241.

Peiró, C. y Devís, J. (1994a) Materiales curriculares y recursos didácticos. En J. Díaz (coord.): Temario de oposiciones al cuerpo de profesores de secundaria, Inde, Barcelona, pp. 11/1-11/18.

Peiró, C. y Devís, J. (1994b) El análisis de materiales curriculares en educación física: un ejemplo. En L. Montero y J.M. Vez (eds.) Las didácticas específicas en la formación del profesorado II (vol. II), Tórculo Edicions, Santiago de Compostela, pp. 775-781.

Placek, J. (1989) An evaluation of the implementation of Basic Stuff fournal of Teaching in Physical Education, 8, 152-161.

Remillard, J. (2000) Can curriculum materiais support teachers' learning? Two fourth-grade teachers' use of a new mathematics text. The Elementary Schoolfournal, 100 (4), 331-350.

Ribas, A. y Guerrero, J.A. (1994) Análisis del sexismo en libros de texto en educación física. En P. Climent; C. Blasco y M $\mathrm{M}^{\mathrm{a}} \mathrm{J}$. López (coords.) La enseñanza de la educación física, Institut Valencia de la Dona, Generalitat Valenciana, Valencia, pp. 379-390.

Rodríguez Diéguez, J.L. etal. (1998) Planes de Estudio y Métodos de Enseñanza (3). Diagnóstico del Sistema Educativo. 1997. (La Escuela Secunadria Obligatoria). MEC. Madrid.

San Martín, A. (1986) Los recursos audiovisuales en el pensamiento pedagógico del profesor. NAU 1libres. Valencia.

San Martín, A. (1991) La organización escolar. Cuadernos de Pedagogía, 194, 26-28.

Santos, M.A. (1991) ¿Cómo evaluar los materiales? Cuadernos de Pedagogía, 194, 29-31.

Schempp, P. (1982) A criticai analysis of the implications in Basic Stuff. fournal of Physical 
Education and Recreation, 53, 20-22.

Tiana, A. (1998) El libro escolar como instrumento didáctico. Concepciones, usos e investigaciones. En A. Escolano (dir.) Historia ilustrada del libro escolar en España. De la posguerra a la reforma educativa, Fundación Germán Sánchez Ruipérez, Madrid, pp. 149-175.

Velasco, M.E. y Pérez, G. (1977) Evaluación y elaboración de textos escolares. Narcea. Madrid.

Venezky, R.L. (1992) Textbooks in school and society. En P.W. Jackson (ed.) Handbook of research on curriculum, Macmillan Pub. Comp., New York, pp. 436-461.

Weinbrenner, P. (1992) Methodologies of Textbooks. Analysis used to date. En H. Bourdillon (ed.) History and Social Studies - Methodologies of Textbooks Analysis. Swets and Zeitlinger. Amsterdam, pp. 2134.

Woodward, A., Elliot, D.L. y Nagel, K.C. (1988) Textbooks in school and society: an annotated bibliography and guide to research. Garland. Nueva York.

Zabala, M.A. (1990) Materiales curriculares. En T. Mauri y otros (coords.) El curriculum en el centro educativo. ICE de la Universitat de Barcelona-Horsori (cuadernos de educación), Barcelona, pp. 125-167. 